J. Electroanal. Chem., 284 (1990) 1-20

Elsevier Sequoia S.A., Lausanne - Printed in The Netherlands

\title{
Membrane potentials of membranes with fixed ionic sites
}

\author{
A. van den Berg a b, P.D. van der Wal a, M. Skowronska-Ptasinska a , \\ E.J.R. Sudhölter ${ }^{a, \star}$, P. Bergveld ${ }^{b}$ and D.N. Reinhoudt ${ }^{a, \star}$ \\ University of Twente, Laboratories of a Organic Chemistry and ${ }^{b}$ Bio-Informatics, P.O. Box 217, \\ 7500 AE Enschede (The Netherlands)
}

(Received 13 July 1989; in revised form 3 November 1989)

\begin{abstract}
A theoretical model has been developed to simulate the formation of a membrane potential as a function of physically accessible parameters. The description is an extension of the well-known TeorellMeyer-Sievers (TMS) model, now including free and fixed ionic sites and free and fixed neutral ligands. The model shows that selectivity loss appears as the molar ratio of [ligand]/[ionic site] $<1$ in the case of fixed ionic sites. The effect of complete immobilization of the neutral ligand has been simulated by gradually decreasing the mobility ratio $\alpha$ of the cation-ligand complex and free cation in the membrane phase. At lower $\alpha$, the membrane potential is reduced by a counteracting diffusion potential. This effect is not present if the product of the association constant $\beta_{1}$, the total ligand concentration $L$ and $\alpha$ is larger than $10^{4}$. Furthermore, it is shown that cation permselectivity of ion-selective membranes that contain fixed anionic sites cannot be eliminated by the incorporation of an equal amount of fixed cationic sites.
\end{abstract}

\section{INTRODUCTION}

Since the first report of an ISFET modified with a valinomycin-containing PVC membrane [1], many improvements have been made in the development of an ISFET-based solid-state ion sensor. These improvements concern better membrane attachment to the ISFET surface by physical [2] or chemical $[3,4]$ methods, suppression of $\mathrm{CO}_{2}$ interference $[5,6]$, and an increased lifetime of the sensor by using a cross-linkable plasticizer [4] or by applying highly lipophilic [7] or even chemically attachable [8] membrane components like ionophores and ionic sites.

However, the question as to whether it is theoretically possible to fabricate a well-functioning ion sensor using immobilized ionic sites and immobilized ionophores in the polymer remains unanswered until now. In order to obtain some

\footnotetext{
ॠ To whom correspondence should be addressed.
} 
insight in the sensing mechanism of such membranes, which is based on the formation of a membrane potential as a function of the composition of the contacting solution, we have developed a model which describes the separate contributions of diffusion and boundary potentials to the total membrane potential. Since we are primarily interested in alkali-cation sensors, the model will be restricted, for the sake of simplicity, to the situation of contacting aqueous solutions that contain only mixtures of monovalent ions.

The model used is principally based on the well-known Teorell-Meyer-Sievers (TMS) theory, which describes the membrane potential for fixed site membranes $[9,10]$. In our description, either free or fixed neutral ligands are also included. Morf [11] showed that for a simplified model, assuming that the contributions of free anions and tight ion-pairs could be neglected, relatively simple analytical expressions can be derived for the membrane potential. The main difference between our description and commonly used models that describe the behaviour of ion-selective membranes is that we have replaced the membrane-confined and hardly mobile, but not fixed, ionic sites by completely immobile (or fixed) sites. The lack of a detailed theoretical description of a system with fixed sites in combination with free or fixed ionophores is probably due to the fact that it has only recently been realized that commonly used PVC membranes can be considered as membranes containing a low concentration of fixed anionic sites [12-14]. Nevertheless, this view was proposed by Perry et al. as early as 1976 [15].

Although qualitative explanations of the ion-sensing mechanism of conventional neutral carrier membranes have been given [11,16-19], only recently has the case of membranes with immobilized ion exchange sites been dealt with to explain the so-called "Donnan exclusion failure" effect [20]. We have considered the operation of polymeric membranes with fixed ionic sites and either free or fixed neutral ligands more generally and have investigated various experimental phenomena in detail.

\section{EXPERIMENTAL}

Potentiometric measurements were performed with PVC- and poly(acrylate)membrane covered ISFETs. The membrane ISFETs were measured with a source and drain follower circuit. The permselective behavior was investigated by measuring the gate voltage of the ISFETs in separate $\mathrm{KCl}$ solutions.

PVC-membrane ISFETs were made by solvent-casting a solution of $1: 2 \mathrm{wt}$ high-molecular-weight PVC (Fluka) and dibutylphthalate (DBP) with varying amounts of di-dodecyldimethylammonium bromide, which resulted in thin films of 5-10 $\mu \mathrm{m}$ thickness.

Poly(acrylate)-membrane ISFETs were made by solvent-casting and photopolymerizing a 1:1 mixture of ACE and DRH 370 (Shell) with 4\% benzophenone. The polymerization was carried out by irradiation with UV light for 2-4 min. Typical layer thicknesses were $2-5 \mu \mathrm{m}$. 
MODEL DESCRIPTION

\section{System definition}

With our model, we can calculate the membrane potential of a membrane which is placed between a sample solution and a reference solution. The sample solution contains an arbitrary mixture of several interfering (secondary) $1: 1$ salts of fixed activity and a varying activity of the primary $1: 1$ salt. The reference solution contains only the primary $1: 1$ salt at a fixed concentration. The cation activities are denoted by $a_{c i, s}$ and $a_{c i, r}$ for the sample and reference solution respectively, with $i=1$ for the primary cations and $i \geqslant 2$ for the secondary cations. By analogy, for the anion activities the symbols $a_{\mathrm{a} i, \mathrm{~s}}$ and $a_{\mathrm{a} i, \mathrm{I}}$ are used. The different fixed anionic or cationic sites in the membrane are indicated by $\mathrm{Y}_{i}^{-}$and $\mathrm{X}_{i}^{+}$, with the corresponding activities $\bar{a}_{\mathrm{Y} i}$ and $\bar{a}_{\mathrm{X} i}$, respectively. The membrane-confined ligand (ionophore) is denoted by $L$, with activity $\bar{a}_{L}$. In general, all parameters in the membrane phase are indicated by an overbar, except for the mobilities $\mu$, since these parameters are only of interest in the membrane phase.

By analogy with the common notation of the membrane potential in the ionselective electrode literature, namely being equal to the potential of the inner reference solution minus the potential of the sample solution, the membrane potential is given by

$E_{\mathrm{m}}=\phi_{\mathrm{r}}-\phi_{\mathrm{s}}$

In order to investigate the properties of a particular membrane composition, the membrane potential response on varying primary ion activity is calculated, contingent on the presence of a fixed activity of secondary ions. In the following sections the calculation procedure is presented.

\section{Diffusion potential}

The diffusion potential $E_{\mathrm{D}}$ is calculated with the use of the Henderson approximation, assuming linear concentration profiles and univalent ions, from the following formula [11]:

$$
\begin{aligned}
E_{\mathrm{D}}=\bar{\phi}_{\mathrm{s}}-\bar{\phi}_{\mathrm{r}}= & \frac{R T}{F} \frac{\sum_{i} \mu_{\mathrm{c} i} \Delta \bar{a}_{\mathrm{c} i}+\sum_{i} \mu_{\mathrm{Lc} i} \Delta \bar{a}_{\mathrm{Lci}}-\sum_{i} \mu_{\mathrm{a} i} \Delta \bar{a}_{\mathrm{a} i}}{\sum_{i} \mu_{\mathrm{c} i} \Delta \bar{a}_{\mathrm{c} i}+\sum_{i} \mu_{\mathrm{Lc} i} \Delta \bar{a}_{\mathrm{Lc} i}+\sum_{i} \mu_{\mathrm{a} i} \Delta \bar{a}_{\mathrm{a} i}} \\
& \times \ln \frac{\sum_{i} \mu_{\mathrm{c} i} \bar{a}_{\mathrm{c} i}(\mathrm{r})+\sum_{i} \mu_{\mathrm{Lci}} \bar{a}_{\mathrm{Lc} i}(\mathrm{r})+\sum_{i} \mu_{\mathrm{a} i} \bar{a}_{\mathrm{a} i}(\mathrm{r})}{\sum_{i} \mu_{\mathrm{c} i} \bar{a}_{\mathrm{c} i}(\mathrm{~s})+\sum_{i} \mu_{\mathrm{Lc} i} \bar{a}_{\mathrm{Lc} i}(\mathrm{~s})+\sum_{i} \mu_{\mathrm{a} i} \bar{a}_{\mathrm{a} i}(\mathrm{~s})}
\end{aligned}
$$

with the following meaning of the symbols:

$\mu_{c i} \quad=$ mobility of cation $i$ in the membrane

$\mu_{\mathrm{a} i} \quad=$ mobility of anion $i$ in the membrane 
$\mu_{\mathrm{Lci}} \quad=$ mobility of ligand-cation complex $i$ in the membrane

$\bar{a}_{\mathrm{LCi}}(\mathrm{s})=$ activity of ligand-cation complex $i$ on the sample side in the membrane

$\bar{a}_{\mathrm{a} i}(\mathrm{~s}) \quad=$ activity of anion $i$ on the sample side in the membrane

$\bar{a}_{\mathrm{c} i}(\mathrm{~s}) \quad=$ activity of cation $i$ on the sample side in the membrane

$\bar{a}_{\mathrm{c} i}(\mathrm{r}) \quad=$ activity of cation $i$ on the reference side in the membrane

$\bar{a}_{\mathrm{a} i}(\mathrm{r})=$ activity of anion $i$ on the reference side in the membrane

$\bar{a}_{\mathrm{Lc} i}(\mathrm{r})=$ activity of ligand-cation complex $i$ on the reference side in the membrane

$\Delta \bar{a}_{\mathrm{c} i} \quad=\bar{a}_{\mathrm{c} i}(\mathrm{r})-\bar{a}_{\mathrm{c} i}(\mathrm{~s})$

$\Delta \bar{a}_{\mathrm{a} i} \quad=\bar{a}_{\mathrm{a} i}(\mathrm{r})-\bar{a}_{\mathrm{a} i}(\mathrm{~s})$

$\Delta \bar{a}_{\mathrm{Lc} i}=\bar{a}_{\mathrm{Lci}}(\mathrm{r})-\bar{a}_{\mathrm{Lci}}(\mathrm{s})$

Although this assumption is incorrect, it has been shown that the errors introduced are small for systems with ions of the same absolute charge [21]. Since the anionic and cationic solution activities are equal, the symbols $a_{\mathrm{c} i}$ and $a_{\mathrm{a} i}$ will be replaced by $a_{i}$ unless clarity requires otherwise. For the computations of $E_{\mathrm{D}}$, relative mobilities can be used (for instance, relative to the primary cation). From eqn. (1) it is obvious that $E_{\mathrm{D}}$ can be calculated if the activities of all (charged) species at both interfaces in the membrane are known. In the next section, it will be shown how the membrane activities of all charged species at a membrane-solution interface can be calculated.

\section{Boundary potential}

For the calculation of a boundary potential $E_{\mathrm{B}}$, which can be written as the difference between the potential $\phi$ in the aqueous phase and $\bar{\phi}$ in the membrane phase, we consider the following equilibrium reactions:

\section{Partition of free ions}

Equilibrium exists between all cations $i$ in the (bulk) membrane phase $\left(\bar{c}_{i}^{+}\right)$and (bulk) aqueous phase $\left(c_{i}^{+}\right)$:

$c_{i}^{+} \stackrel{k_{\mathrm{c} i}}{\rightleftharpoons} \bar{c}_{i}^{+}$

The partition coefficient $k_{\mathrm{c} i}$ can be derived from the identity of the electrochemical potentials in the aqueous phase $\left(\tilde{\mu}_{c i}\right)$ and membrane phase $\left(\overline{\tilde{\mu}}_{\mathrm{c} i}\right)$ at equilibrium:

$\tilde{\mu}_{c i}=\overline{\tilde{\mu}}_{\mathrm{c} i}$

which is equivalent to:

$\mu_{\mathrm{c} i}^{\circ}+R T \ln a_{\mathrm{c} i}+F \phi=\bar{\mu}_{\mathrm{c} i}^{\circ}+R T \ln \bar{a}_{\mathrm{c} i}+F \bar{\phi}$

where $\mu_{\mathrm{c} i}^{\circ}$ is the standard chemical potential of cation $i$. If the activity coefficient is assumed to be unity, the partition coefficient $k_{\mathrm{c} i}$ can be defined as the ratio $\bar{a}_{\mathrm{c} i} / a_{\mathrm{c} i}$ 
in the absence of a boundary potential, which gives an expression for $k_{\mathrm{c} i}$ from eqn. (2):

$\left.k_{\mathrm{c} i}\right|_{E_{\mathrm{B}}=0}=\bar{a}_{\mathrm{c} i} / a_{\mathrm{c} i}=\exp \left(\left(\mu_{\mathrm{c} i}^{\circ}-\bar{\mu}_{\mathrm{c} i}^{\circ}\right) / R T\right)$

This means that the partition coefficient is a chemical parameter, fully determined by the difference in standard chemical potentials between the two phases. In the presence of a boundary potential, the ratio $\bar{a}_{\mathrm{c} i} / a_{\mathrm{c} i}$ is simply expressed as

$\bar{a}_{\mathrm{c} i} / a_{\mathrm{c} i}=k_{\mathrm{ci}} \times \exp \left(-F E_{\mathrm{B}} / R T\right)$

For anions, the partition coefficient is defined in a similar way, and consequently the anion activities in the aqueous and membrane phases are related by

$\bar{a}_{\mathrm{a} i} / a_{\mathrm{a} i}=k_{\mathrm{a} i} \times \exp \left(F E_{\mathrm{B}} / R T\right)$

\section{Complexation of free cations}

Every cation in the membrane can be complexed by free ligand molecules:

$\mathrm{L}+\bar{c}_{i}^{+} \stackrel{\beta_{i}}{\rightleftharpoons} \mathrm{L} \cdot \overline{\mathrm{c}}_{i}^{+} \quad \beta_{i}=\bar{a}_{\mathrm{L} \mathrm{c} i} /\left(\bar{a}_{\mathrm{c} i} \times \bar{a}_{\mathrm{L}}\right)$

in which $\beta_{i}$ is the association constant of the complex ligand with cation $i$.

Association of anions with fixed cationic sites

Fixed cationic sites may form ion-pairs with free anions in the membrane according to

$\mathrm{X}_{i}^{+}+\bar{a}_{j}^{-} \stackrel{K_{\mathrm{X} i j}}{\rightleftharpoons} \mathrm{X}_{i} \cdot \overline{\mathrm{a}}_{j} \quad K_{\mathrm{X} i j}=\bar{a}_{\mathrm{X} i \mathrm{a} j} /\left(\bar{a}_{\mathrm{a} j} \times \bar{a}_{\mathrm{X} i}\right)$

in which $K_{\mathrm{X}_{i j}}$ is the ion-pair formation constant of cationic site $\mathrm{X}_{i}^{+}$with anion $j$.

Association of free and complexed cations with fixed anionic sites

Fixed anionic sites may form ion-pairs both with free cations in the membrane and with ligand-cation complexes. The equilibrium for ion-pairing of free cations gives

$\mathrm{Y}_{i}^{-}+\overline{\mathrm{c}}_{j}^{+} \stackrel{K_{\mathrm{Y}_{i j}}}{\rightleftharpoons} \mathrm{Y}_{i} \cdot \overline{\mathrm{c}}_{j} \quad \mathrm{~K}_{\mathrm{Y}_{i j}}=\bar{a}_{\mathrm{Yicj}} /\left(\bar{a}_{\mathrm{c} j} \times \bar{a}_{\mathrm{Y} i}\right)$

in which $K_{\mathrm{Y}_{i j}}$ is the ion-pair formation constant of anionic site $\mathrm{Y}_{i}^{-}$with cation $j$, and the association of ligand-cation complexes can be described by

$\mathrm{Y}_{i}^{-}+\mathrm{L} \cdot \overline{\mathrm{c}}_{j}^{+} \stackrel{K_{\mathrm{L}}}{\rightleftharpoons} \mathrm{Y}_{i} \cdot \mathrm{L} \cdot \overline{\mathrm{c}}_{j} \quad K_{\mathrm{L}}=\bar{a}_{\mathrm{Y} i \mathrm{Lc} j} /\left(\bar{a}_{\mathrm{Lc} j} \times \bar{a}_{\mathrm{Yi}}\right)$

In eqn. (9), only one association constant $K_{\mathrm{L}}$ is used for all the different anionic site-ligand-cation complexes, $\mathrm{Y}_{i} \cdot \mathrm{L} \cdot \mathrm{c}_{j}$, in order to avoid mathematical complications. However, in most practical cases there will be a significant concentration of complexes only with the primary ions, and therefore this approximation seems to be justified. Furthermore, in solving the final set of equations, it appears necessary to consider only one type of anionic site. 
Besides the equilibria mentioned above, conservation equations can be obtained for the immobile ionic sites and ligand if their activity coefficients are taken as unity.

\section{Conservation of cationic sites}

For every cationic site $\mathrm{X}_{i}$, it holds that the sum of the activity of ionized sites $\mathrm{X}_{i}^{+}$ and the activity of ion-paired sites with any anion $j, \mathrm{X}_{i} \cdot \bar{a}_{j}$, equals the total activity of cationic sites $X_{i, \text { tot }}$ :

$\bar{a}_{\mathrm{X} i}+\sum_{j} \bar{a}_{\mathrm{X} i \mathrm{aj}}=X_{i, \mathrm{ot}}$

where the summation is taken over all free anions $j$.

Conservation of anionic sites

For the anionic sites $Y_{i}$ the situation is more complicated, because not only the association of the sites with all free cations $j$ has to be taken into account, but also the association with ligand-cation complexes, $\mathrm{L} \cdot \overline{\mathrm{c}}_{j}^{+}$:

$\bar{a}_{\mathrm{Yi}}+\sum_{j} \bar{a}_{\mathrm{Yicj}+} \sum_{j} \bar{a}_{\mathrm{Y} i \mathrm{Lc} j}=Y_{i, \text { tot }}$

Of course, in this case the summation is taken over all different cations $j$.

\section{Conservation of ligand}

The last equation of conservation concerns the total activity of ligand molecules in the membrane:

$\bar{a}_{\mathrm{L}}+\sum_{i} \bar{a}_{\mathrm{Lci}}+\sum_{i} \sum_{j} \bar{a}_{\mathrm{Y} j \mathrm{Lc} i}=L_{\mathrm{tot}}$

In eqn. (12) a double summation has to be taken, namely, over the complexes with all the different cations $i$, and their ion-pairs with all the different anionic sites $j$.

\section{Electroneutrality of the bulk membrane}

The partition equations (4) and (5), association equations (6)-(9), and conservation equations (10)-(12) provide nine equations for ten unknown variables $\left(a_{\mathrm{a} i}, a_{\mathrm{c} i}\right.$, $\bar{a}_{\mathrm{Y} i}, \bar{a}_{\mathrm{Yicj}}, \bar{a}_{\mathrm{Y} i \mathrm{Lcj}}, \bar{a}_{\mathrm{X} i}, \bar{a}_{\mathrm{X} i \mathrm{a} j}, \bar{a}_{\mathrm{L}}, \bar{a}_{\mathrm{L} c i}$ and $\left.E_{\mathrm{B}}\right)$. This means that one more equation is needed in order to solve $E_{\mathrm{B}}$ from this set. Apart from the space charge regions, the membrane remains electrically neutral ${ }^{\star}$. Since equilibrium reactions between the bulk of the membrane and the bulk of the aqueous solutions are considered, it is reasonable to add the condition of electroneutrality to the equations mentioned above:

$\Sigma$ all cationic species $+\sum$ all anionic species $=0$

Substitution of the nine previous equations into eqn.(13) leaves, after some straight-

\footnotetext{
* Aguilella et al. showed that the excess charge and ion concentration in the diffusion layer differ by almost nine orders of magnitude under typical conditions (see ref. 22).
} 
forward mathematics, one equation, which generally gives an implicit solution for $E_{\mathrm{B}}$. Accordingly, an iterative procedure is applied to calculate $E_{\mathrm{M}}$ (see Appendix).

\section{Total membrane potential}

If $E_{\mathrm{B}}$ is known, the activities of all the cations and anions in the membrane, which are needed to determine $E_{\mathrm{D}}$ from eqn. (1), can be calculated. Thus, the total membrane potential $E_{\mathrm{M}}=E_{\mathrm{Bs}}-E_{\mathrm{D}}-E_{\mathrm{Br}}$ can be evaluated. It should be emphasized that this model is very universal: for every ionic species a particular mobility, partition coefficient, fixed-site association constant and (in the case of cations) complexation constant can be specified. Even the system of a membrane with membrane-confined relative immobile ions can be simulated by providing the sample solution with a fixed concentration of very lipophilic ions with a small relative mobility. On the other hand, rather simple systems can be mimicked by setting the appropriate parameters to zero or unity. In the next section some calculated results obtained with the model will be presented.

\section{RESULTS AND DISCUSSION}

The model was used to investigate three different issues, namely:

(1) the behaviour of ion-selective membranes;

(2) the measurements with PVC and acrylate-type membranes; and

(3) the effect of immobilization of ligands.

Illustration of basic properties and special phenomena in ion-selective membranes

\section{Permselectivity}

The (cation) permselectivity was simulated using the model by taking a fixed anionic site concentration of $10^{-3} \mathrm{M}$ in the membrane, and equal anion and cation mobilities and partition coefficients. The results observed are depicted in Fig. 1a. It is clear that the observed Nernstian permselectivity is casued by a boundary potential, as expected. In Fig. $1 \mathrm{~b}$, the loss of permselectivity occurring at high salt activities is shown as a function of a decreasing fixed-site concentration (curves 1-5). Increasing the ion-pair formation constant $K_{Y}$ appears to have the same effect (curve 6 vs. curve 1).

In the second case, the (cation) permselectivity was simulated by the mobility differences of the extracted ions, by taking zero sample anion mobility and no fixed sites in the membrane. The results are shown in Fig. 2 . The origin of the observed permselectivity (Fig. 2a) shows up as a diffusion potential, as expected. The dependence of the potentiometric slope in the permselective region on the mobility ratio $\mu_{\mathrm{a}} / \mu_{\mathrm{c}}$ is illustrated in Fig. $2 \mathrm{~b}$.

Loss of selectivity

A well-known phenomenon of ion-selective membranes is the loss of selectivity which occurs when the ligand leaches out of the membrane. For membranes 

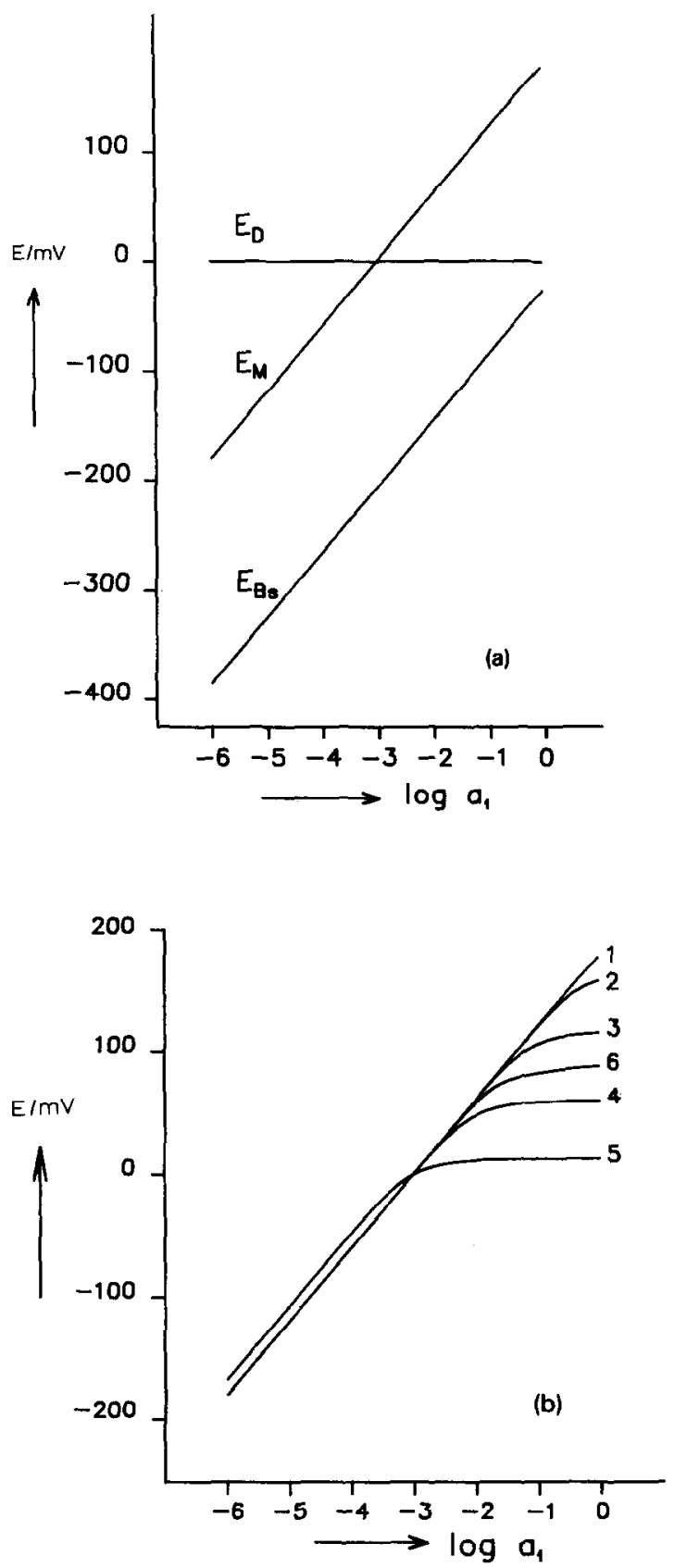

Fig. 1. Calculated membrane potential response on primary ion activity. Parameters: (a) $\mu_{a} / \mu_{c}=1$; $a_{\text {ref }}=10^{-3} \mathrm{M} ; K_{\mathrm{Y}}=10^{4} \mathrm{M}^{-1}, k_{\mathrm{a}}=k_{\mathrm{c}}=10^{-4} ; Y_{\mathrm{tot}}=10^{-3} \mathrm{M} ; L_{\mathrm{tot}}=0 \mathrm{M}$; (b) see (a), except for curves 1-5: $Y_{\text {tot }}=10^{-3}, 10^{-4}, 10^{-5}, 10^{-6}, 10^{-7} \mathrm{M}$, and for curve 6: $Y_{\text {tot }}=10^{-3} \mathrm{M} ; K_{\mathrm{Y}}=10^{8} \mathrm{M}^{-1}$. 

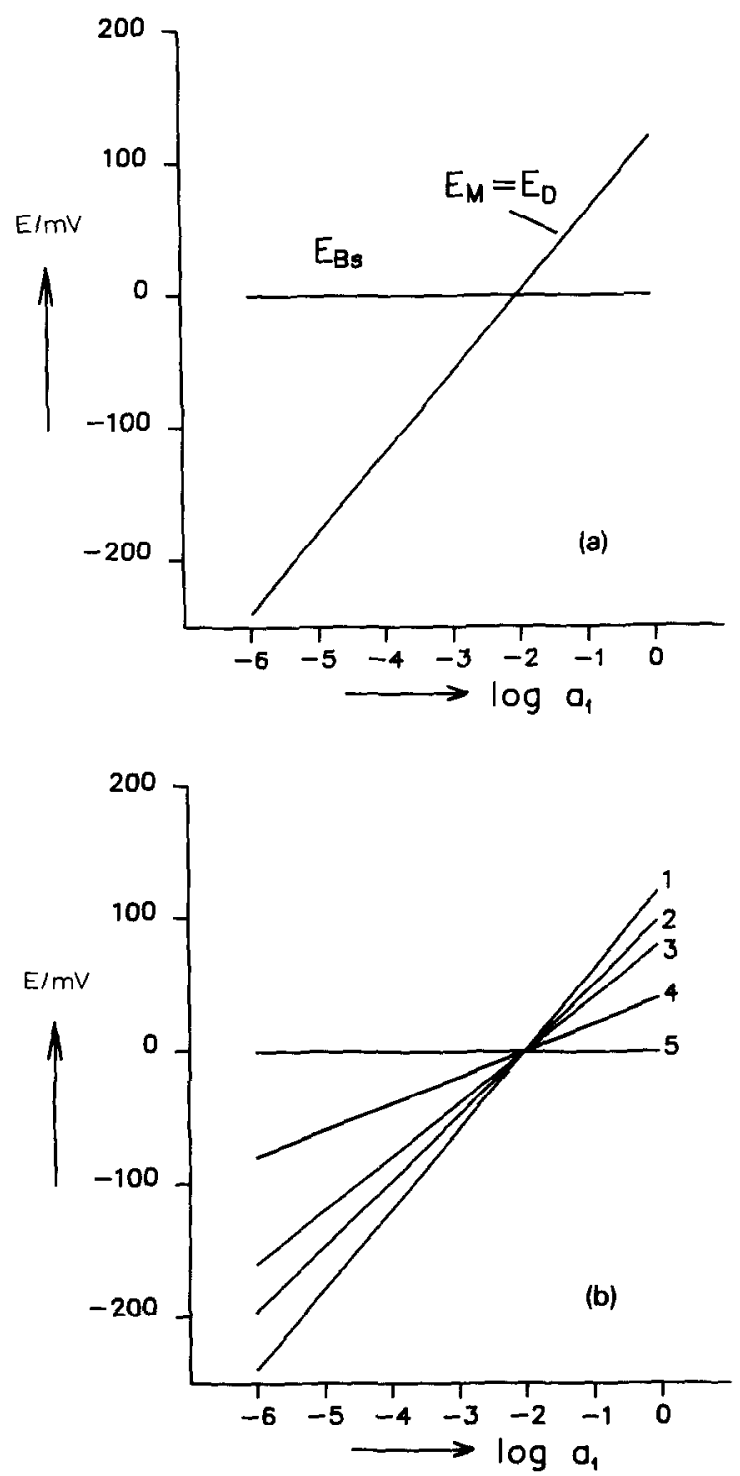

Fig. 2. Calculated membrane potential response on primary ion activity. Parameters: (a) $\mu_{\mathrm{a}} / \mu_{\mathrm{c}}=0$; $k_{\mathrm{a}}=k_{\mathrm{c}}=10^{-4} ; K_{\mathrm{Y}}=10^{4} \mathrm{M}^{-1} ; a_{\text {ref }}=10^{-2} \mathrm{M} ; Y_{\text {tot }}=0 \mathrm{M} ; L_{\mathrm{tot}}=0 \mathrm{M} ;$ (b) see (a), except for curves $1-5: \mu_{\mathrm{a}} / \mu_{\mathrm{c}}=0,0.1,0.2,0.5$ and 1 , respectively.

containing anionic sites with low, but non-zero mobility it has been shown that the critical ratio [ligand]/[ionic sites] $\simeq 1$ [23]. In Fig. 3 the same effect is demonstrated in the case of fixed sites. In this figure, the response of a membrane loaded with ligand and fixed anionic sites on the primary ion activity is shown, in the presence 


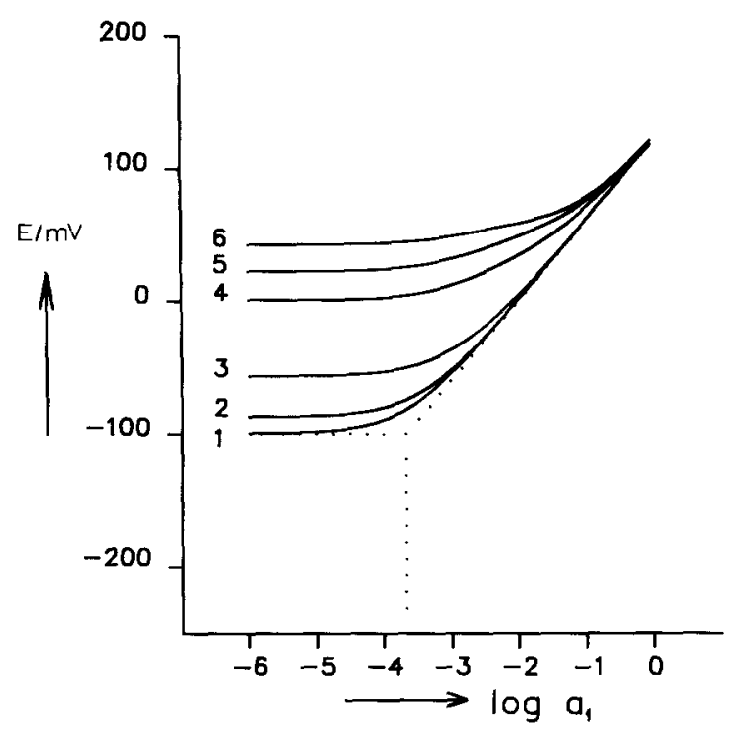

Fig. 3. Illustration of selectivity loss in a membrane loaded with fixed anionic sites and ligand. Parameters: see Fig. 1, and: $Y_{\text {tor }}=10^{-3} \mathrm{M} ; a_{2}=10^{-1} \mathrm{M} ; \beta_{1}=10^{5} \mathrm{M}^{-1} ; \beta_{2}=10^{2} \mathrm{M}^{-1}$; curves 1-6: $L_{\text {tot }}=10^{-2}, 5 \times 10^{-3}, 2 \times 10^{-3}, 10^{-3}, 8 \times 10^{-4}$ and $5 \times 10^{-4} \mathrm{M}$, respectively.

of interfering ions at $0.1 \mathrm{M}$. In curves 1-6 the ratio [ligand]/[fixed sites] varies from 10 to 0.5 , and the corresponding loss of selectivity is evidently demonstrated. An interesting aspect is the difference between curves 2 and 1 , which indicates that this ratio should actually not just be larger than one, but as large as possible. Even the selectivity calculated from curve $1\left(\log k_{i j}=-2.7\right)$ does not quite reflect the ratio of the association constants taken: $\beta_{1} / \beta_{2}=10^{3}$. Nevertheless, the slope of the response is practically Nernstian in the sensitive region.

\section{Donnan exclusion failure (DEF)}

The presence of lipophilic sample anions is the most trivial reason for the observation of the Donnan exclusion failure effect. This situation is simulated in Fig. 4. When the partition coefficient for anions, $k_{\mathrm{a}}$, is increased from $10^{-5}$ to 10 (curves 1-4), the cationic response at low activities changes into an anionic response at high sample ion activities. From Fig. 4 the linear relation between the position of the maximum in the curve $\left(\log (a)_{\max }\right)$ and $\log \left(k_{\mathrm{a}}^{1 / 2}\right)$ can be observed. Other simulations verify that the relation between $\log (a)_{\max }$ and the ligand concentration, fixed-site concentration, cation and anion partition parameters and association constant can be written as

$\log (a)_{\max } \propto \log \left(k_{\mathbf{a}}^{1 / 2} k_{\mathbf{c}}^{1 / 2} \beta^{1 / 2} L_{\text {tot }}^{1 / 2}\right)$

This was stated previously by Buck et al. [20].

This means that the best way to eliminate this effect is to ensure a high 


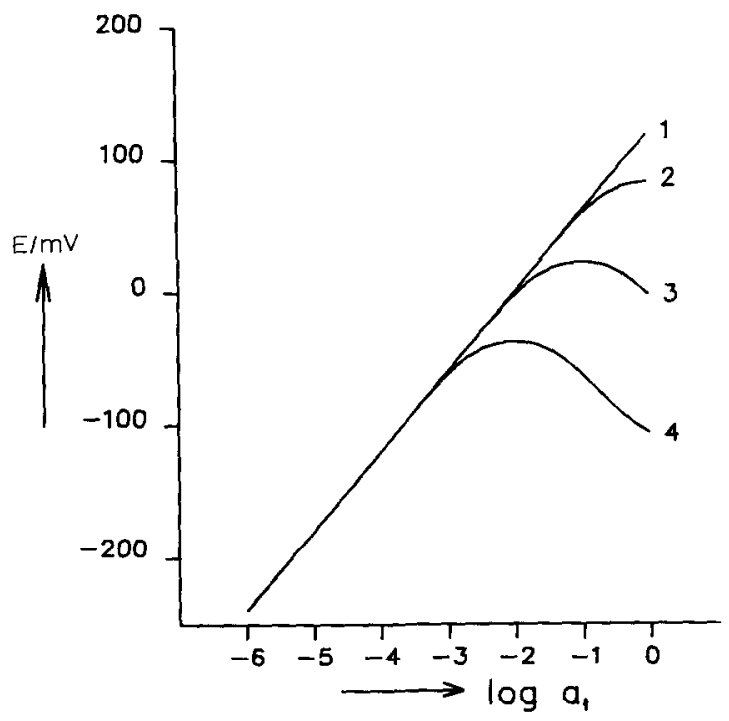

Fig. 4. Illustration of Donnan exclusion failure for a membrane loaded with fixed anionic sites and ligand. Parameters: see Fig. 1 , and: $k_{\mathrm{c}}=10^{-5} ; L_{\mathrm{tot}}=10^{-2} \mathrm{M} ; Y_{\mathrm{tot}}=10^{-3} \mathrm{M}$; curves 1-4: $k_{\mathrm{a}}=10^{-5}$, $10^{-3}, 10^{-1}$ and 10 .

concentration of fixed sites in the membrane, analogous to the solution in the case of anionic sites with low mobility.

Potentiometric measurements with PVC and acrylate-type polymers

In our work concerning the development of an integrated ion sensor system, the development of a reference-FET (REFET) as an integral part is an essential element $[24,25]$. Ideally, the polymeric membranes applied on REFETs should have a membrane potential which is independent of the solution composition. In practice, one of the most important requirements, namely insensitivity to $\mathrm{H}^{+}$ions, is easily fulfilled under normal conditions $\left(a_{\mathrm{H}}<a_{\mathrm{c}}, a_{\mathrm{OH}}<a_{\mathrm{a}}\right)$. However, the permselective behaviour of most polymeric membranes appears to give an undesired ionic strength dependency of the membrane potential, and thus of the REFET signal. Therefore lipophilic cations were added to both a PVC membrane and an acrylate membrane in order to reduce this effect. The results are summarized in Fig. 5. Quaternary ammonium tetraphenyl borate has been added to valinomycin-containing plasticized PVC membranes in order to reduce the membrane resistance [26].

In our experiments, a gradual change in the permselective behaviour was found upon the addition of hardly mobile cations. Since our concept is based on the application of polymers with fixed membrane components, it was interesting to see whether this behaviour could also be anticipated with polymers containing both 

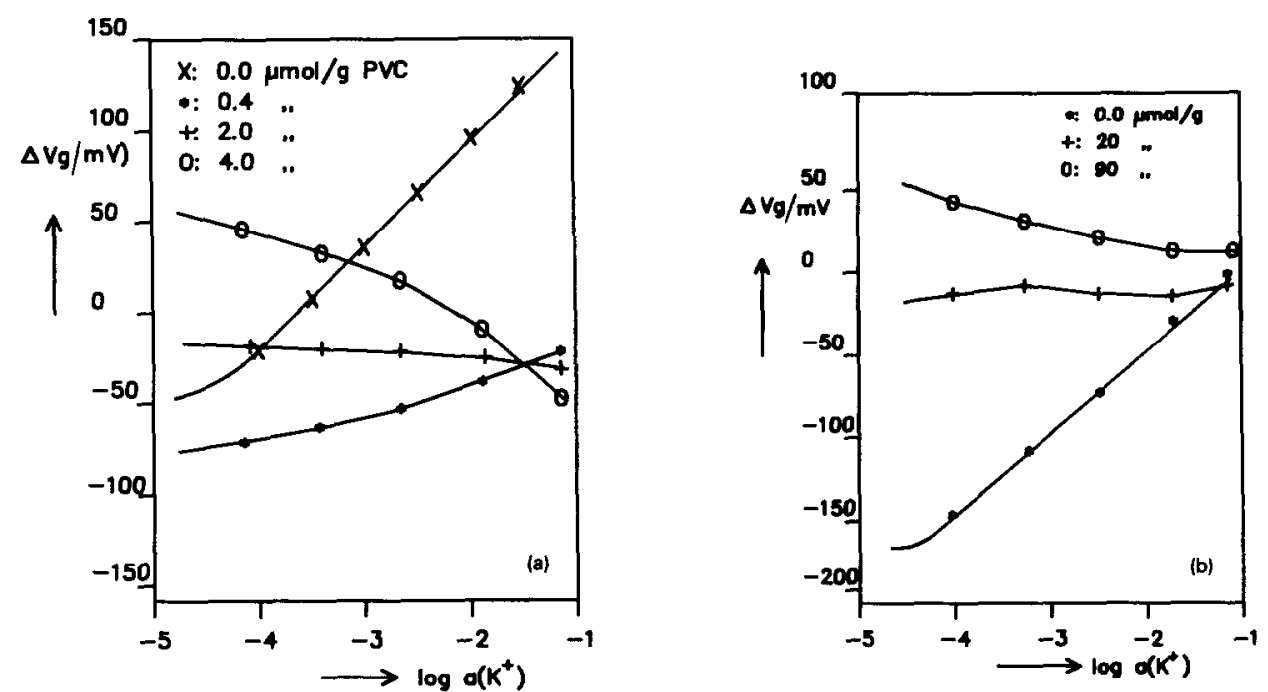

Fig. 5. Measured effect of the addition of $\mathrm{DDMA}^{+}$ions on the permselectivity of (a) PVC and (b) an acrylate REFET.

negative and positive fixed ionic sites. Therefore model calculations were carried out for such systems with model parameters such that extraction of sample ions did not contribute significantly to the total charge in the membrane; if so, a diffusion potential depending on the ratio of the anion and cation mobilities would be encountered. It appeared that, unlike the results shown in Fig. 5, in the case of fixed sites a very sudden change in permselectivity occurred at the ratio $\bar{a}_{\mathrm{X}} / \bar{a}_{\mathrm{Y}}=1$ (see Fig. 6).

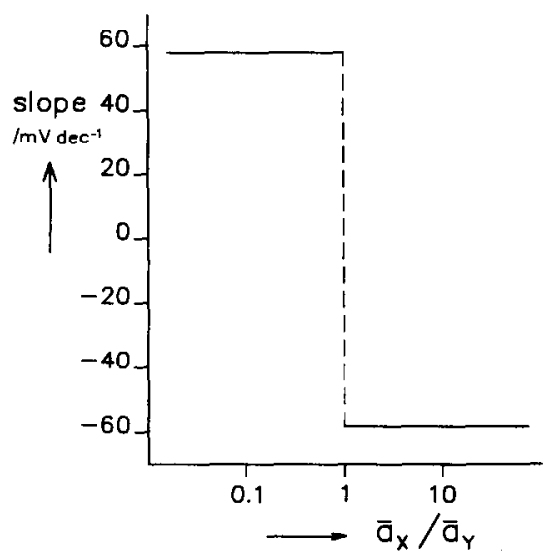

Fig. 6. Calculated potentiometric slope of the membrane potential as a function of the sample ion activity for different $a_{\mathrm{X}} / a_{\mathrm{Y}}$ ratios. 
This result did not depend on the differences between the anion and cation mobilities or the partition coefficients. This result is in agreement with the recent results of Yamauchi et al. [27], who investigated the behaviour of an amphoteric ion-exchange membrane containing $0.192 \mathrm{mmol} / \mathrm{g}-\mathrm{SO}_{3}^{-}$sites as well as 0.183 mmol $/ \mathrm{g}-\mathrm{C}_{5} \mathrm{H}_{4} \mathrm{~N}^{+}\left(\mathrm{CH}_{3}\right)_{3}$ sites, in contact on both sides with aqueous solutions. In spite of the relatively small excess of positively charged groups, a perfect Nernstian anion response for both $\mathrm{NaCl}$-containing solutions and solutions which contained much more lipophilic cations like tetramethylammonium and tetraethylammonium was found.

From the experimental and simulated results, the following conclusions can be drawn:

(1) The gradual change in permselectivity change of PVC and polyacrylate with ionic strength is caused by a diffusion potential, which suggests the presence of ions with low mobility in the membranes.

(2) Amphoteric ion-exchange membranes cannot be applied directly for REFET purposes.

A solution could be to use two REFETs in a differential arrangement, both covered with an ion-exchange membrane. One of the ion-exchange membranes should contain fixed anionic sites and the other one fixed cationic sites. The sum of the two membrane potentials, one Nernstian anionic and the other Nernstian cationic, should be independent of the ionic strengths, and, at high site densities, the influence of lipophilic sample ions can also be excluded.

\section{The application of fixed ligands}

In principle, there are two different ways to analyse the behaviour of membranes with both fixed sites and fixed ligand molecules. The first one is to consider the membrane as an ion-exchange membrane, with fixed sites that have different association constants for different ions. Morf [11] showed that theoretically no selective Nernstian response is to be expected in such a system. This finding would, in his view, explain the failure of experiments carried out with negatively charged ionophores like nigericin and monensin [28]. Unfortunately, he did not describe the mechanism of this failure. For that reason, we have considered the behaviour of a membrane with fixed sites and ligand from another point of view. Using the model with mobile ligands and taking the parameters in Fig. 3 (curve 1), we decreased the mobility ratio $\mu_{\mathrm{Lc} i} / \mu_{\mathrm{c} i}$. In this way, we simulated the effect of an immobilization procedure. As a result, a lower membrane potential response upon the primary ion activity was calculated (at constant activity of the interfering ion), which was caused by the development of a counteracting diffusion potential (see Fig. 7). The polarity of this diffusion potential was opposite to that of the boundary potential. An intriguing question now is the origin of this diffusion potential. If the expression for the diffusion potential in eqn. (1) is considered, and it is further assumed that anionic contributions to the diffusion potential are negligible owing to the very low free 


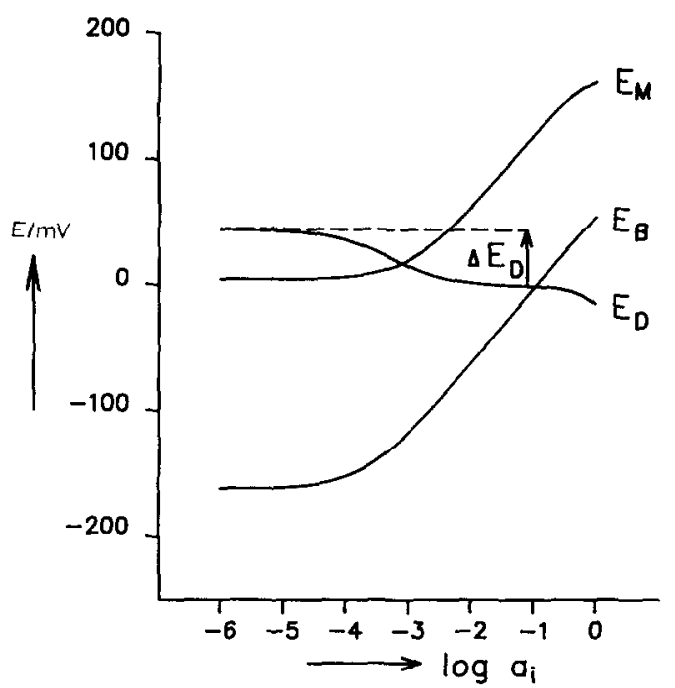

Fig. 7. Calculated selectivity loss due to immobilization of the ionophore parameters: see Fig. 3, curve 1, except for $\mu_{\mathrm{Lc} i}=\mu_{c i} / 10$.

anion concentration, we obtain for the diffusion potential

$E_{\mathrm{D}}=\frac{R T}{F} \times \ln \frac{\sum_{i} \mu_{\mathrm{c} i} \bar{a}_{\mathrm{c} i}(\mathrm{r})+\sum_{i} \mu_{\mathrm{Lci} i} \bar{a}_{\mathrm{Lc} i}(\mathrm{r})}{\sum_{i} \mu_{\mathrm{c} i} \bar{a}_{\mathrm{c} i}(\mathrm{~s})+\sum_{i} \mu_{\mathrm{Lc} i} \bar{a}_{\mathrm{Lc} i}(\mathrm{~s})}$

with the summation carried out over all cationic species. We take as a measure for the counteracting diffusion potential the difference in its value at primary ion activities of $10^{-4}$ and $10^{-1} \mathrm{M}$. Thus, we have to calculate

$$
\begin{aligned}
\Delta E_{\mathrm{D}}= & E_{\mathrm{D}}\left(10^{-4}\right)-E_{\mathrm{D}}\left(10^{-1}\right) \\
= & \frac{R T}{F} \times \ln \frac{\sum_{i} \mu_{\mathrm{c} i} \bar{a}_{\mathrm{ci} i}(\mathrm{r})+\sum_{i} \mu_{\mathrm{Lc} i} \bar{a}_{\mathrm{Lc} i}(\mathrm{r})}{\sum_{\mathrm{ci}} \bar{a}_{\mathrm{ci}}\left(10^{-4}\right)+\sum_{i} \mu_{\mathrm{Lci} i} \bar{a}_{\mathrm{Lc} i}\left(10^{-4}\right)} \\
& -\frac{R T}{F} \times \ln \frac{\sum_{i} \mu_{\mathrm{c} i} \bar{a}_{\mathrm{c} i}(\mathrm{r})+\sum_{i} \mu_{\mathrm{Lc} i} \bar{a}_{\mathrm{Lc} i}(\mathrm{r})}{\sum_{i} \mu_{\mathrm{c} i} \bar{a}_{\mathrm{c} i}\left(10^{-1}\right)+\sum_{i} \mu_{\mathrm{Lc} i} \bar{a}_{\mathrm{Lc} i}\left(10^{-1}\right)} \\
= & \frac{R T}{F} \times \ln \frac{\sum_{i} \mu_{\mathrm{c} i} \bar{a}_{\mathrm{c} i}\left(10^{-1}\right)+\sum_{i} \mu_{\mathrm{Lc} i} \bar{a}_{\mathrm{Lc} i}\left(10^{-1}\right)}{\sum_{i} \mu_{\mathrm{c} i} \bar{a}_{\mathrm{c} i}\left(10^{-4}\right)+\sum_{i} \mu_{\mathrm{Lci}} \bar{a}_{\mathrm{Lc} i}\left(10^{-4}\right)}
\end{aligned}
$$

If the summations are elaborated for cations and complexes 1 and 2 , and if the 
primary ion activities $10^{-1}$ and $10^{-4} \mathrm{M}$ are denoted by indices $\mathrm{h}$ and 1 respectively, the following relation is obtained:

$\Delta E_{\mathrm{D}}=\frac{R T}{F} \times \ln \frac{\sum_{i} \mu_{\mathrm{cl}} \bar{a}_{\mathrm{cl}}(\mathrm{h})+\mu_{\mathrm{c} 2} \bar{a}_{\mathrm{c} 2}(\mathrm{~h})+\mu_{\mathrm{Lc} 1} \bar{a}_{\mathrm{Lc} 1}(\mathrm{~h})+\mu_{\mathrm{Lc} 2} \bar{a}_{\mathrm{Lc} 2}(\mathrm{~h})}{\sum_{i} \mu_{\mathrm{c} 1} \bar{a}_{\mathrm{cl}}(1)+\mu_{\mathrm{c} 2} \bar{a}_{\mathrm{c} 2}(1)+\mu_{\mathrm{Lc1}} \bar{a}_{\mathrm{Lc} 1}(1)+\mu_{\mathrm{Lc} 2} \bar{a}_{\mathrm{Lc} 2}(1)}$

From eqn. (17) we can discuss why, under conditions of a low complex mobility, a diffusion potential builds up. It should be noted that under conditions of equal cation and ligand-cation complex mobilities, the activity gradients of the cationic species over the membrane do not result in a diffusion potential, since the total amount of positive charge must counterbalance the fixed negative sites activity, which is constant. Under normal circumstances, almost all the positive charge is formed by the complexes, and the product $\mu_{\mathrm{Lcl}} \bar{a}_{\mathrm{Lcl}}$ is the most important term in eqn. (17). However, if the mobility of the complex decreases, and if the product $\mu_{\mathrm{c} 1} \bar{a}_{\mathrm{c} 1}$ becomes comparable to $\mu_{\mathrm{Lcl}} \bar{a}_{\mathrm{Lcl}}$ (which is approximately equal to $\left.\mu_{\mathrm{Lc1}} \beta_{1} L_{\mathrm{tot}} \bar{a}_{\mathrm{cl}}\right)$, the free cations also contribute to the diffusion potential. In Fig. $8 \mathrm{a}$ the formation of a diffusion potential $E_{\mathrm{D}}$ is shown as a function of the mobility ratio $\mu_{\mathrm{Lcl}} / \mu_{\mathrm{cl}}=\alpha$.

It appears that three regions can be distinguished: a region without a significant diffusion potential, which occurs at large $\alpha$ values; a linear region, where variations in the mobility ratio immediately lead to a change in the diffusion potential; and a saturation region at very low $\alpha$ values. As expected, an influence of $\beta_{1}$ and $L_{\text {tot }}$ is also found: curves 2 and 3 in Fig. 8a show the "delay" in the onset of diffusion potential formation in the case of increased ligand activity. From Fig. $8 \mathrm{~b}$ it follows that increasing the association constant has a similar effect. From the latter figure, it is also clear that the curves shift by one $\log (\alpha)$ unit in the direction of lower $\alpha$ values upon increasing $\beta_{1}$ by a factor of 10 . A comparable effect would be expected in Fig. 8a. However, in Fig. 8b we changed the partition coefficients from $10^{-4}$ to $10^{-7}$, in order to exclude the extraction of free ions, which occurs relatively easily with the higher $\beta_{1}$ value. Since in Fig. 8a we applied the original partition parameters from Fig. 3, in this case some influence from the free cations and anions was experienced. From the results we are able to define a value for the product $\alpha \beta_{1} L_{\text {tot }}$, which indicates the start of the counteracting diffusion potential development. The arrows in Fig. 8 indicate that a choice of $\alpha \beta_{1} L_{\text {tot }}=10^{4}$ gives good discrimination between good and bad functioning of such a system.

\section{CONCLUSIONS}

In this paper we have illustrated the mechanism of potential formation in fixed-site membranes with or without neutral ligands. A model was presented to calculate the membrane potential of such systems, and we showed that several well-known phenomena could be explained and interpreted. Moreover, it was shown 

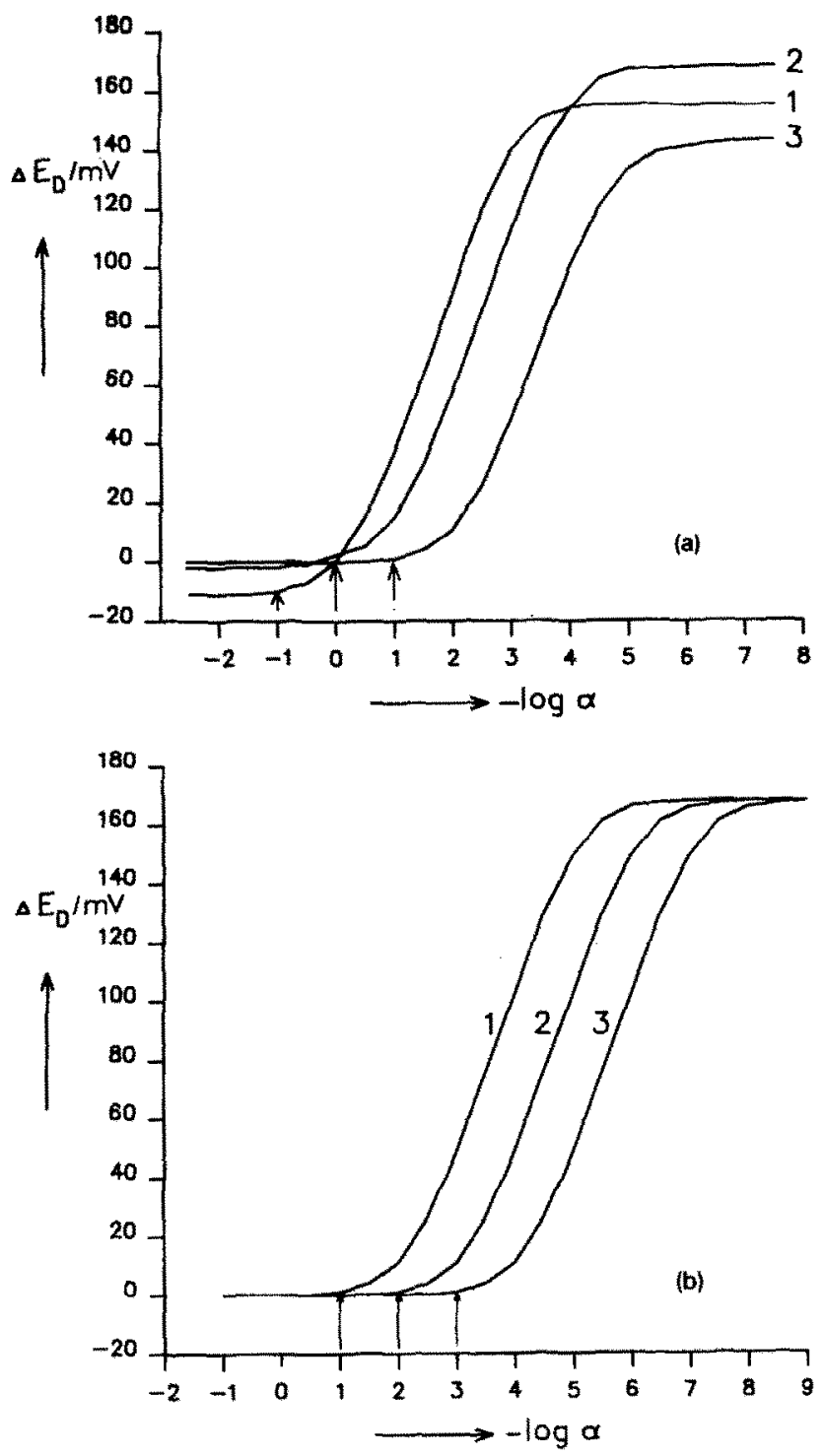

Fig. 8. Calculated diffusion potential build-up as a function of the complex/cation mobility ratio $\alpha$. Parameters: (a) see Fig. 3, curve 1. Curve 2: $L_{\text {tot }}=10^{-1} \mathrm{M}, Y_{\mathrm{tot}}=10^{-2} \mathrm{M}$. Curve 3: $L_{\mathrm{tot}}=1 \mathrm{M}$, $Y_{\text {tot }}=10^{-2} \mathrm{M}$. (b) Curves 1-3, with $k_{\mathrm{a} i}=k_{\mathrm{cl}}=10^{-7} \mathrm{M}^{-1}$, and $\beta_{1}=10^{7} \mathrm{M}^{-1}, \beta_{2}=10^{4} \mathrm{M}^{-1}$.

that the condition that the ligand to anionic site ratio is larger than one, which is valid for mobile-site membranes, also holds for fixed-site membranes.

Application of the model to investigate the behaviour of membranes with added lipophilic cations showed that only with mobile sites can insensitivity to ionic 
strength be expected. This means that for the development of a REFET fixed-site membranes cannot be applied simply.

Investigation of a system with both fixed anionic sites and an immobilized ligand led to the conclusion that complete immobilization of ligand molecules results in a lower membrane potential, when compared with the situation with mobile ligand molecules. It was found that this effect was caused by the development of a counteracting diffusion potential. Thus, an immobilization procedure of ligands, for instance by attaching them to the polymer backbone, should at least be accompanied by the provision of some kind of flexibility of the ligand, which gives them a small effective mobility although they are fixed to the polymer. Apart from this, a high association constant of the ligand used and a high ligand concentration suppress the mentioned effect.

\section{ACKNOWLEDGEMENTS}

The Centre of Microelectronics (CME) Twente is gratefully acknowledged for financial support.

\section{APPENDIX}

Starting with eqn. (13), there are three contributions to the positive charges and two contributions to the negative charges. First the positive charges are considered:

(1) Free cations

$\sum_{i=1}^{n_{\mathrm{s}}} \bar{a}_{\mathrm{c} i}=\sum_{i=1}^{n_{\mathrm{s}}} k_{\mathrm{c} i} a_{i} \exp \left(-F E_{\mathrm{B}} / R T\right)$

where $a_{i}$ is the solution cation (anion) activity of salt $i$ and $n_{\mathrm{s}}$ is the number of $1: 1$ salts. If we write $x=\exp \left(-F E_{\mathrm{B}} / R T\right)$, we obtain

$$
\sum_{i=1}^{n_{\mathrm{s}}} \bar{a}_{\mathrm{c} i}=x \sum_{i=1}^{n_{\mathrm{s}}} k_{\mathrm{c} i} \times a_{i}
$$

(2) Not "ion-paired" cationic sites

For every cationic site $\mathrm{X}_{i}$, it holds that

$$
\bar{a}_{\mathbf{X} i}=X_{i, \text { tot }}-\bar{a}_{\mathbf{X}_{i}} \sum_{i=1}^{n_{\mathrm{s}}} k_{\mathrm{X}_{i j}} \times a_{j}
$$

where $a_{j}$ is the cation (anion) solution activity of salt $j$. Equation (A3) can be written as

$$
\bar{a}_{\mathrm{X} i}=X_{i, \mathrm{ot}} /\left(1+\sum_{j=1}^{n_{\mathrm{s}}} K_{\mathrm{X} i j} \times a_{j}\right)
$$


Thus, for all cationic sites $i$

$$
\sum_{j=1}^{n_{\mathrm{x}}} \bar{a}_{\mathrm{X} i}=\sum_{i=1}^{n_{\mathrm{x}}}\left(\frac{X_{i, \mathrm{tot}}}{\left(1+\sum_{j=1}^{n_{\mathrm{s}}} K_{\mathrm{X} i j} \times a_{j}\right)}\right)
$$

where $n_{\mathrm{x}}$ is the number of cationic fixed sites.

\section{(3) Ligand-cation complexes}

From eqns. (6) and (12) we obtain for the total amount of ligand-cation complexes

$$
\sum_{i=1}^{n_{\mathrm{s}}} \bar{a}_{\mathrm{Lc} i}=\left(L_{\mathrm{tot}}-\sum_{i}^{n_{\mathrm{s}}} \bar{a}_{\mathrm{Lc} i}-\sum_{i}^{n_{\mathrm{s}} n_{\mathrm{y}}} \bar{a}_{\mathrm{Y} j \mathrm{~L} c i}\right) \sum_{i}^{n_{\mathrm{s}}} \beta_{i} \bar{a}_{\mathrm{c} i}
$$

from this with the help of eqn. (9) we have

$$
\sum_{i=1}^{n_{\mathrm{s}}} \bar{a}_{\mathrm{Lc} i}=\frac{L_{\mathrm{tot}} \sum_{i}^{n_{\mathrm{s}}} \beta_{i} \bar{a}_{\mathrm{c} i}}{1+\sum_{i}^{n_{\mathrm{s}}} \beta_{i} \bar{a}_{\mathrm{c} i}+K_{\mathrm{L}} \sum_{j}^{n_{\mathrm{y}}} \bar{a}_{\mathrm{Y} j} \sum_{i}^{n_{\mathrm{s}}} \beta_{i} \bar{a}_{\mathrm{c} i}}
$$

With

$$
\begin{aligned}
& X X=\sum_{i=1}^{n_{\mathrm{s}}} \bar{a}_{\mathrm{Lc} i} \\
& C=K_{\mathrm{L}} \sum_{i}^{n_{\mathrm{s}}} \beta_{i} \bar{a}_{\mathrm{c} i} \\
& D=L_{\mathrm{tot}} \sum_{i}^{n_{\mathrm{s}}} \beta_{i} \bar{a}_{\mathrm{c} i} \\
& E=1+\sum_{i}^{n_{\mathrm{s}}} \beta_{i} \bar{a}_{\mathrm{c} i} \\
& Y Y=\sum_{j}^{n_{\mathrm{Y}}} \bar{a}_{\mathrm{Y} j}
\end{aligned}
$$

we are able to write eqn. (A7) as

$X X=\frac{D}{E+C \times \bar{Y} Y}$

For negative charges we have similar equations: 
(4) Free anions

$\sum_{i=1}^{n_{\mathrm{s}}} \bar{a}_{\mathrm{a} i}=\sum_{i}^{n_{\mathrm{s}}} k_{\mathrm{a} i} a_{i} \exp \left(F E_{\mathrm{B}} / R T\right)=x \sum_{i}^{n_{\mathrm{s}}} k_{\mathrm{a} i} \times a_{i}$

(5) Not "ion-paired" anionic sites

From eqns. (8) and (9) we obtain for the total concentration of free anionic sites $i$ :

$\bar{a}_{\mathrm{Y} i}=Y_{i, \mathrm{tot}}-\bar{a}_{\mathrm{Y} i} \sum_{j}^{n_{\mathrm{s}}} K_{\mathrm{Y} i j} \bar{a}_{\mathrm{c} j}-\bar{a}_{\mathrm{Y} i} \sum_{j}^{n_{\mathrm{s}}} K_{\mathrm{L}} \bar{a}_{\mathrm{Lc} j}$

which leads to

$\bar{a}_{\mathrm{Y} i}=Y_{i, \mathrm{tot}} /\left(1+\sum_{j}^{n_{\mathrm{s}}} K_{\mathrm{Y} i j} \bar{a}_{\mathrm{c} j}+K_{\mathrm{L}} \sum_{j}^{n_{\mathrm{s}}} \bar{a}_{\mathrm{Lc} j}\right)$

In order to be able to add up the contributions of all the different sites $Y_{i}$, and to eliminate the term $\sum_{i=1}^{n_{s}} \bar{a}_{L c i}$ from eqns.(A7) and (A11), only one single ion-pair formation constant has to be assumed for all the anionic sites $i$, thus $K_{Y_{i j}}=K_{Y_{J} \text {. }}$. Now we obtain

$\sum_{i}^{n_{\mathrm{y}}} \bar{a}_{\mathrm{Y} i}=\sum_{i}^{n_{\mathrm{y}}} Y_{i, \mathrm{tot}} /\left(1+\sum_{j}^{n_{\mathrm{s}}} K_{\mathrm{Y} j} \bar{a}_{\mathrm{c} j}+K_{\mathrm{L}} \sum_{j}^{n_{\mathrm{s}}} \bar{a}_{\mathrm{Lc} j}\right)$

which can be written as

$\sum_{i}^{n_{\mathrm{y}}} \bar{a}_{\mathrm{Y} i}=Y Y=\frac{A}{B+K_{\mathrm{L}} \times X X}$

where

$A=\sum_{i}^{n_{y}} Y_{i, \text { ot }}$

$B=1+\sum_{j}^{n_{\mathrm{s}}} K_{\mathrm{Y} j} \bar{a}_{\mathrm{c} j}$

and $X X$ is as mentioned before. Now $X X$ and $Y Y$ can be calculated from the combination of eqns. (A13) and (A8). If eqns. (A2), (A5), (A7), (A9) and (A12) are combined, an implicit expression in $x$ is obtained of the form

$F(x)=0$

It can be shown that for large $x$, and $x$ approaching to zero, the function $F(x)$ has an opposite sign. Moreover, since this equation reflects a physical system, there is only one value for $x$ for which $F(x)$ equals zero. An iterative procedure provides the required solution for $x$, which can be converted into the boundary potential. 


\section{REFERENCES}

1 S.D. Moss, J. Janata and C.C. Johnson, Anal. Chem., 47 (1975) 2238.

2 H. Blennemann, L. Bousse and J.D. Meindl in Proceedings Transducers '87, Tokyo, 1987, p. 723.

3 E.J.R. Sudhölter, P.D.van der Wal, M. Skowronska-Ptasinska, A. van den Berg and D.N. Reinhoudt, Sensors Actuators, 17 (1989) 189.

4 D.J. Harrison, A. Teclemariam and L.L. Cunningham in ref. 2, p. 763.

5 E.J.R. Sudhölter, P.D. van der Wal, M. Skowronska-Ptasinska, A. van den Berg, P. Bergveld and D.N. Reinhoudt, Anal. Chim. Acta, 230 (1990) 59.

6 D.J. Harrison, L. Xizhong and E.M.J. Verpoorte in ref. 2, p. 738.

7 U. Oesch, W. Simon, Anal. Chem.,52 (1980) 692.

8 A.G. Talma, H. van Vossen, E.J.R. Sudhölter, J. van Eerden and D.N. Reinhoudt, Synthesis, 8 (1986) 680.

9 T. Teorell, Proc. Soc. Exp. Biol. Med., 33 (1935) 282.

10 K.H. Meyer and J.F. Sievers, Helv. Chim. Acta, 19 (1936) 649.

11 W.E. Morf, The Principles of Ion-Selective Electrodes and of Membrane Transport, Elsevier, Amsterdam, 1981.

12 W.E. Morf and W. Simon, Helv. Chim. Acta, 69 (1986) 1120.

13 G. Horvai, E. Graf, K. Toth, E. Pungor and R.P. Buck, Anal. Chem., 58 (1986) 2735.

14 A. van de Berg, P.D. van der Wal, M. Skowronska-Ptasinska, E.J.R. Sudhölter and D.N. Reinhoudt, Anal. Chem., 59 (1987) 2827.

15 M. Perry, E. Löbel and R. Bloch, J. Membr. Sci., 1(1976) 223.

16 J.D.R. Thomas, Anal. Chim. Acta, 180 (1986) 289.

17 R. Büchi, E. Pretsch, W.E. Morf and W. Simon, Helv. Chim. Acta, 59 (1976) 2407.

18 D.C. Crawley and G.A. Rechnitz, J. Membr. Sci., 24 (1985) 201.

19 R.D. Armstrong, A.K. Covington and G.P. Evans, J. Electroanal. Chem., 159 (1983) 33.

20 R.P. Buck, K. Toth, E. Graf, G. Horvai and E. Pungor J. Electroanal. Chem., 223 (1987) 51.

21 R.P. Buck in H. Freiser (Ed.), Ion Selective Electrodes in Analytical Chemistry, Vol. 1, Plenum Press, New York, 1978, Ch. 1.

22 V.M. Aguilella, S. Mafé and J. Pellicer, Electrochim. Acta, 32 (1987) 483.

23 P.C. Meier, W.E. Morf, M. Laubli and W. Simon, Anal. Chim. Acta, 156 (1984) 1.

24 M. Skowronska-Ptasinska, P.D. van der Wal, A. van den Berg, P. Bergveld, E.J.R. Sudhölter and D.N. Reinhoudt, Anal. Chim. Acta, 230 (1990) 67.

25 P. Bergveld, A. van den Berg, P.D. van der Wal, M. Skowronska-Ptasinska, E.J.R. Sudhölter and D.N. Reinhoudt, Sensors Actuators, 18 (1989) 309.

26 G. Horvai, T.A. Nieman and E. Pungor, 4th Symposium on Ion Selective Electrodes, Matrafüred, 1984, p. 439.

27 A. Yamauchi, Y. Okazaki, R. Kurosaki, Y. Hirata and H. Kimizuka, J. Membr. Sci., 32 (1987) 281.

28 ORION Research Inc., Newsletter, 2 (1970) 14. 